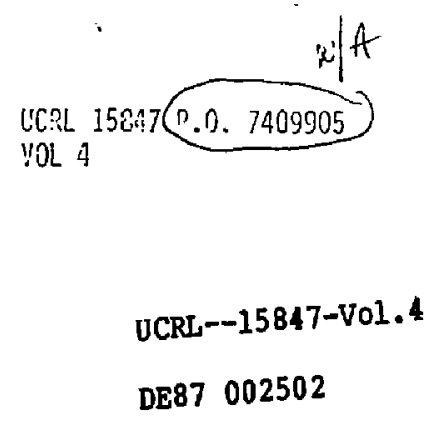
CALCULATED FISSION PMOPEPTIES
OF THE HENUIEST ELE:!E:ITS
VIL 4
PAIPINI, I!! !UUCLE]

P. Iloller, 1. Iix, i. Suiatecki

Senteriber 1986

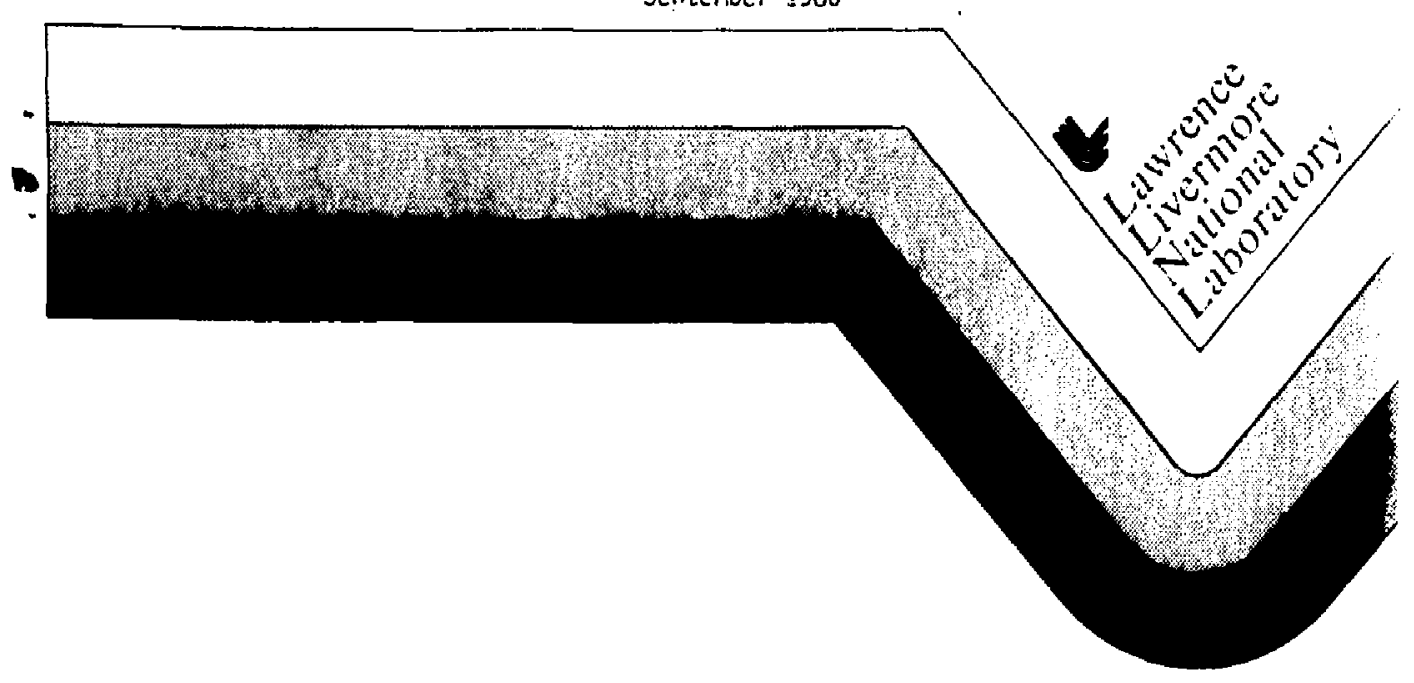


DISCLANEk

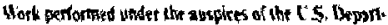
ment of Enero by lawrence tisermatr Nutional lebort16r: endea contust: number W-705-EXG-48.

This doneratm wes prepared is in acroust of work

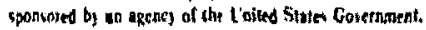

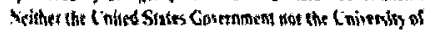

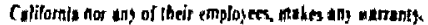

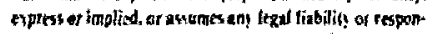

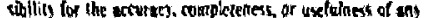
informaliose appsralus produch of process dixtosed. of reprosents that its use nould oot infriste prikately onned

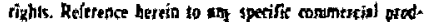
ons, proness at cervire by inade name, indematk, manofic"

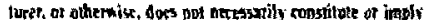
its endarsemest, recotomendation ar fausting by the thited

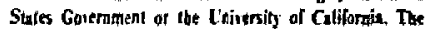

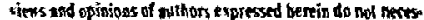

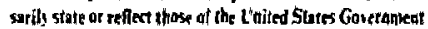

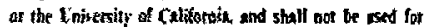
aducrising or prod uet endorkenent purposes. 


\title{
Pairing in Nuclei
}

\author{
P. Möller \\ Lawrence Livermore National Laboratory, Livermore, CA 94550 \\ D. G. Madland and J. R. Nix
}

Theoretical Division, Los Alamos National Laboratory, Los Alamos, NM 87545

April 3, 1986

New expressions for the nuclear neutron and proton average pairing gaps have been derived by ref.[1]. They find

$$
\bar{\Delta}_{\mathrm{n}}=\frac{c B_{\mathrm{i}}}{N^{1 / s}} e^{-a I-b I^{2}}
$$

and

$$
\bar{\Delta}_{\mathrm{p}}=\frac{c B_{1}}{Z^{1 / s}} e^{+a J-b I^{2}}
$$

with

$$
I=\frac{N-Z}{N+Z}
$$

Here $I$ is the relative neutron excess and $B_{0}$ is the ratio of the surface area of the nucleus at the deformation studied to the surface area of the spherical nucleus. The three constants $a, b$ and $c$ are determined by a least-squares adjustment to experimental pairing gaps obtained from measured masses. In addition ref.|1] introduced a new expression for the average residual n-p interaction energy $\delta$ appearing in the masses of odd-odd nuclei. They suggest

$$
\delta=\frac{d}{A^{2 / 3}}
$$

Preliminary values for the parameters of this model have been determined with the approximation that $B_{\mathrm{a}}=1$ for all nuclei. The values obtained are $a=0.097, b=7.97$, $c=5.75 \mathrm{MeV}$ and $d=6.71 \mathrm{MeV}$.

We have used this model as input into a calculation of nuclear masses according to step $B 7$ in the research plan, section 'GROUND-STATE MATSES AND SINGLEPARTICLE LEVELS'. Relative to step B5 there was no change in the two first significant digits of the calculated rms deviations. The nuclear mass model contains as one part a microscopic pairing model, which is discussed in ref.[2]. Thus our calculation also yields proton and neutron pairing $\Delta$ 's calculated in this model.

In fig. 1a we show the proton pairing gap derived from measured masses plotted with different symbols depending on the magnitude of the calculated ground-state deformation. In addition we show the average pairing gap according to the present model as a solid line and also, as a dashed line, the proton average pairing gap under the assumption $\bar{\Delta}_{\mathrm{p}}=12 / \sqrt{A} \mathrm{MeV}$. Figure $1 \mathrm{~b}$ is similar, except that here the symbols correspond to proton pairing gaps calculated in the BCS approximation. In fig. 1c we show the difference between the pairing gap determined from experimental masses and 
the pairing gap calculated from the BCS equations. Figs. 2a-2c show the corresponding quantities for neutrons. The solid and dashed lines in figs. $1 \mathrm{a}, 1 \mathrm{~b}, 2 \mathrm{a}$ and $2 \mathrm{~b}$ are calculated for for elements along the valley of beta stability.

From the results displayed in the figures one can observe the following. For protons the new model for the average pairing gap agrees better with the data than the old $\left(\bar{\Delta}_{\mathrm{p}}=12 / \sqrt{A} \mathrm{MeV}\right)$ model. For neutrons there is no major difference between the models for $\bar{\Delta}_{\mathrm{n}}$, but far from stability there would be larger differences. Another observation is that the calculated values for $\Delta_{p}$ and $\Delta_{n}$ are usually below the curve for $\bar{\Delta}$, in particular for the heavier systems. This can be understood by the following argument [3]. In our pairing calculation the pairing strength $G$ is determined from the relationship that exists between $G$ and the average pairing gap $\bar{\Delta}$ for a uniform distribution of levels[2]. This pairing strength is then used to calculate the pairing energy from a set of ealculated single-particle levels corresponding to the nucleus studied.

However, for a uniform energy level distribution the pairing can act more strongly than across the gap in the level spectrum that is usually present at the ground state of a nucleus. When $G$ is determined from the uniform level distribution, where the pairing acts strongly, a relatively small $G$ results for a particular value of $\bar{\Delta}$. When this $G$ is used in a calculation with a nonuniform level distribution corresponding to an actual nucleus a smaller $\Delta$ results. It is clear that the model should be somewhat modified to avoid this systematic error.

A third difficulty occurs near magic numbers. Here figs $1 c$ and $2 c$ show large discrepancies between theory and calculations. This may be partly due to errors in the $\Delta$ 's determined from experiment, because they are difficult to extract from mass regions where the deformation changes rapidly. However, the largest source of the discrepancy is probably that the BCS approximation collapses for large gaps in the single-particle spectrum. Also close to the region of collapse the solutions to the BCS equations are not very accurate.

We intend to study improvements to the pairing model, with the aim to remove both the systematic underestimate of the pairing $\Delta$ 's and the difficulties associated with the collapse close to magic numbers.

\section{References}

[1] D. G. Madland and J. R. Nix, Bull. Am. Phys. Soc. to appear.

[2] M. Bolsterli, E. O. Fiset, J. R. Nix and J. L. Norton, Phys. Rev. C5 (1972) 1050.

[3] T. Døssing and A. S. Jensen, Nucl. Phys. A222 (1974) 493. 


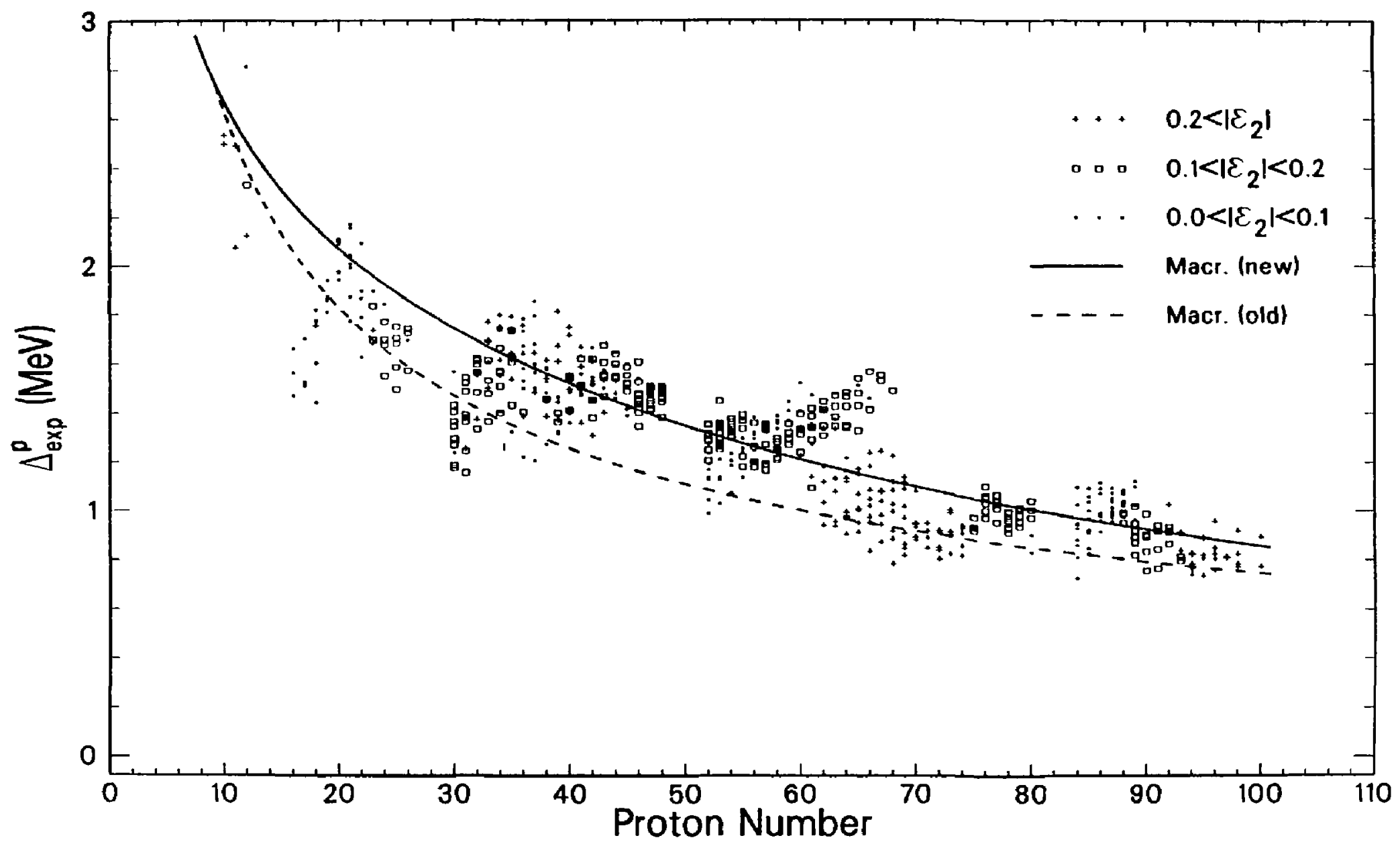

Fig. la 


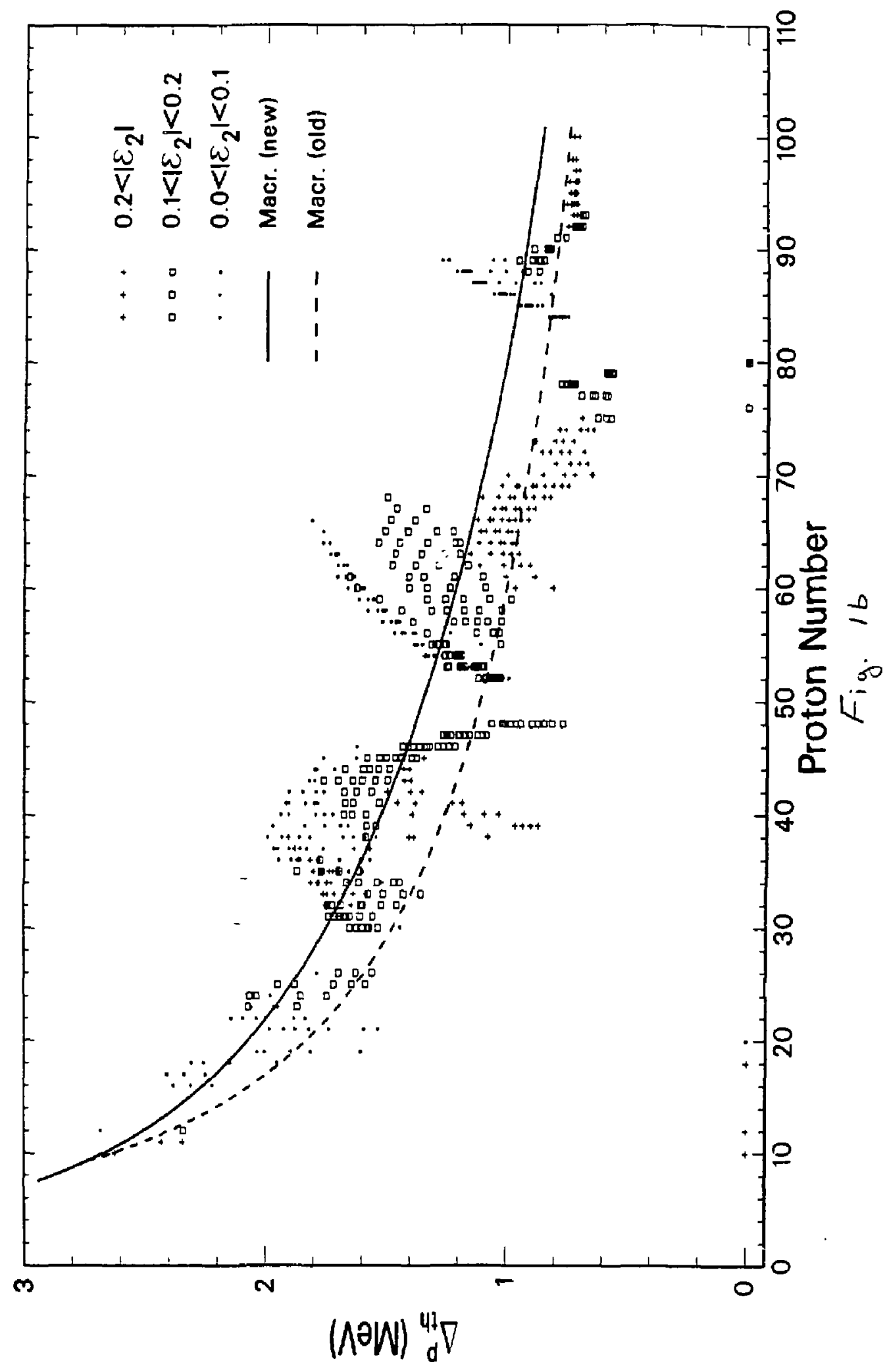




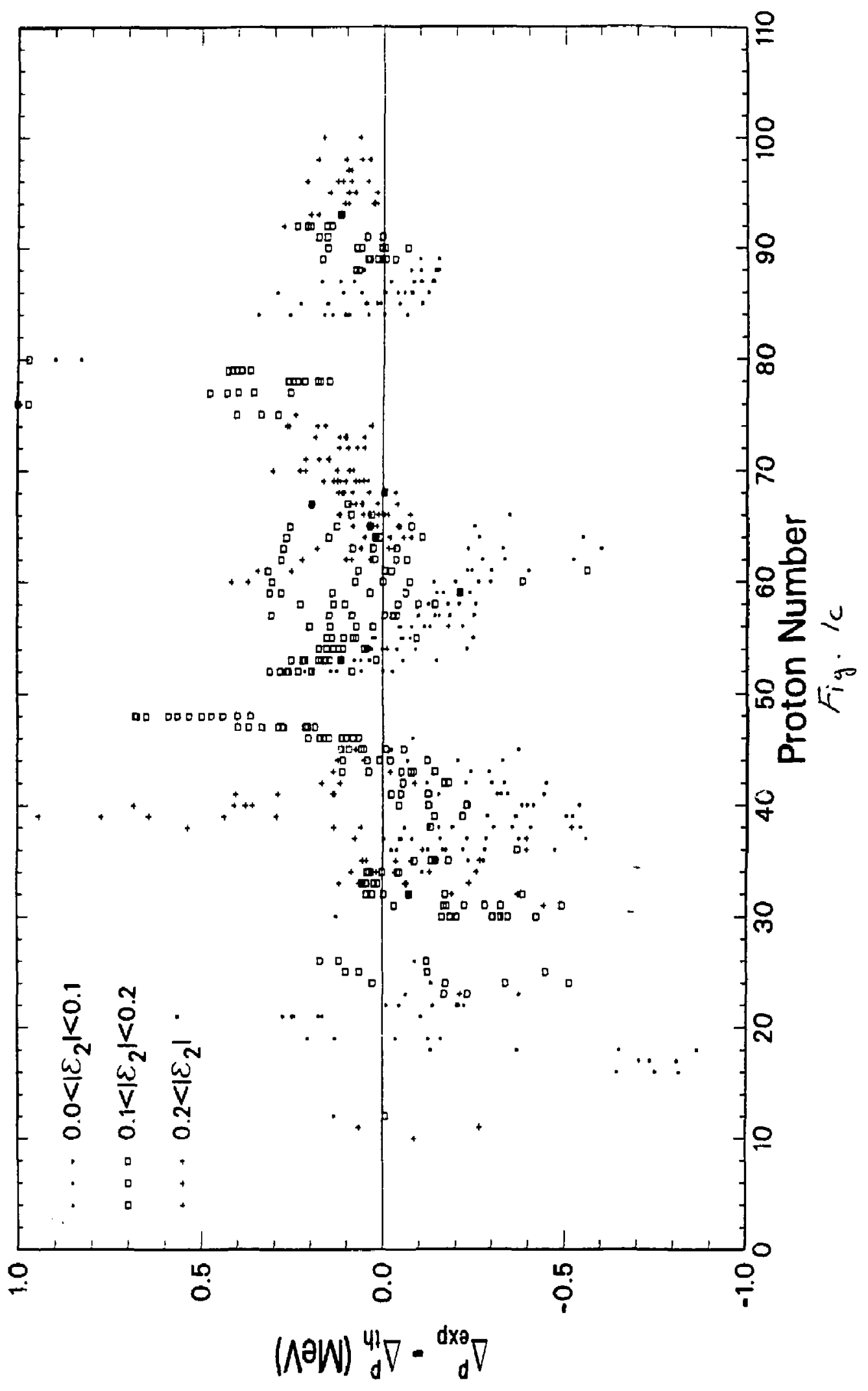




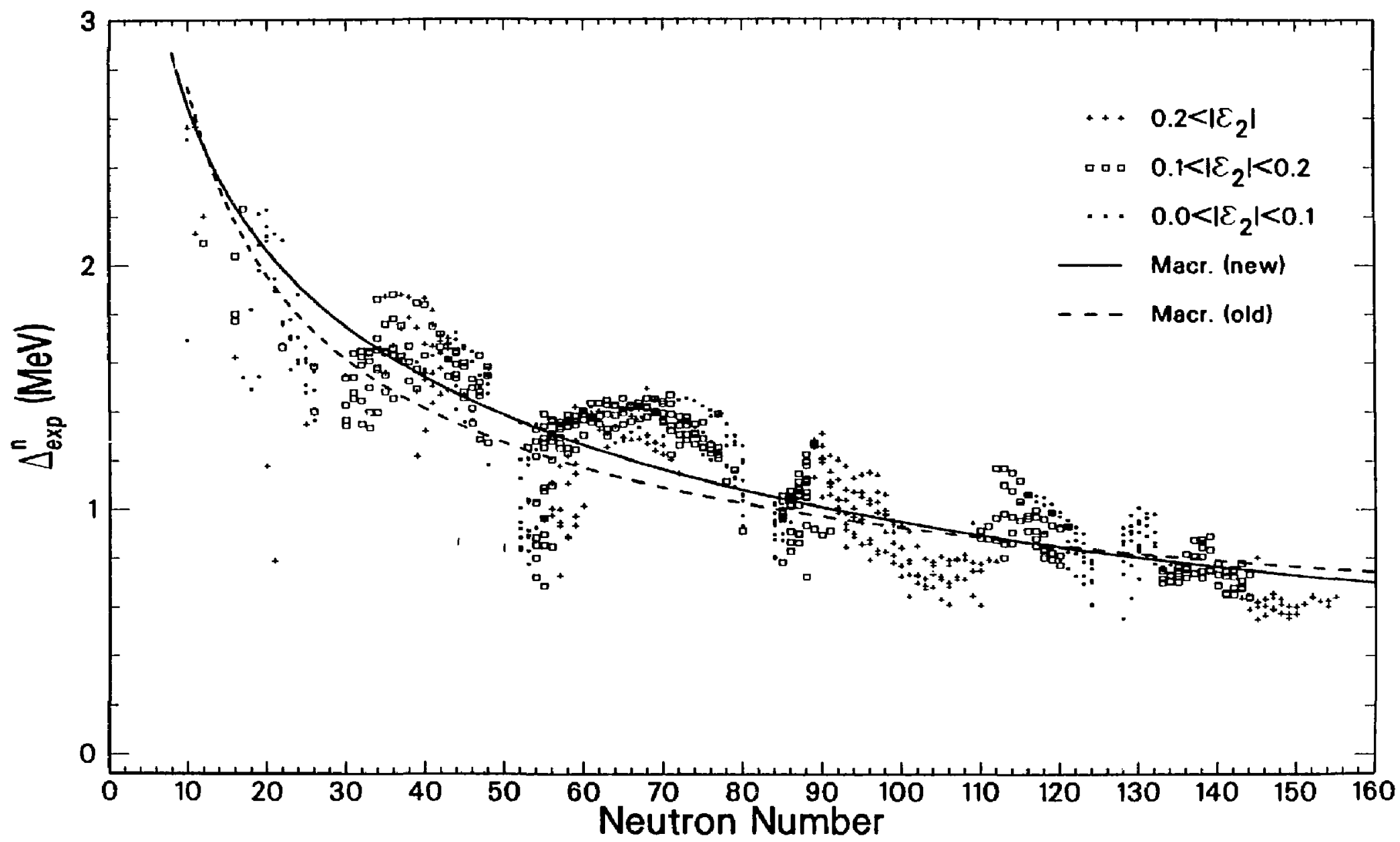

Fig. $2 a$ 


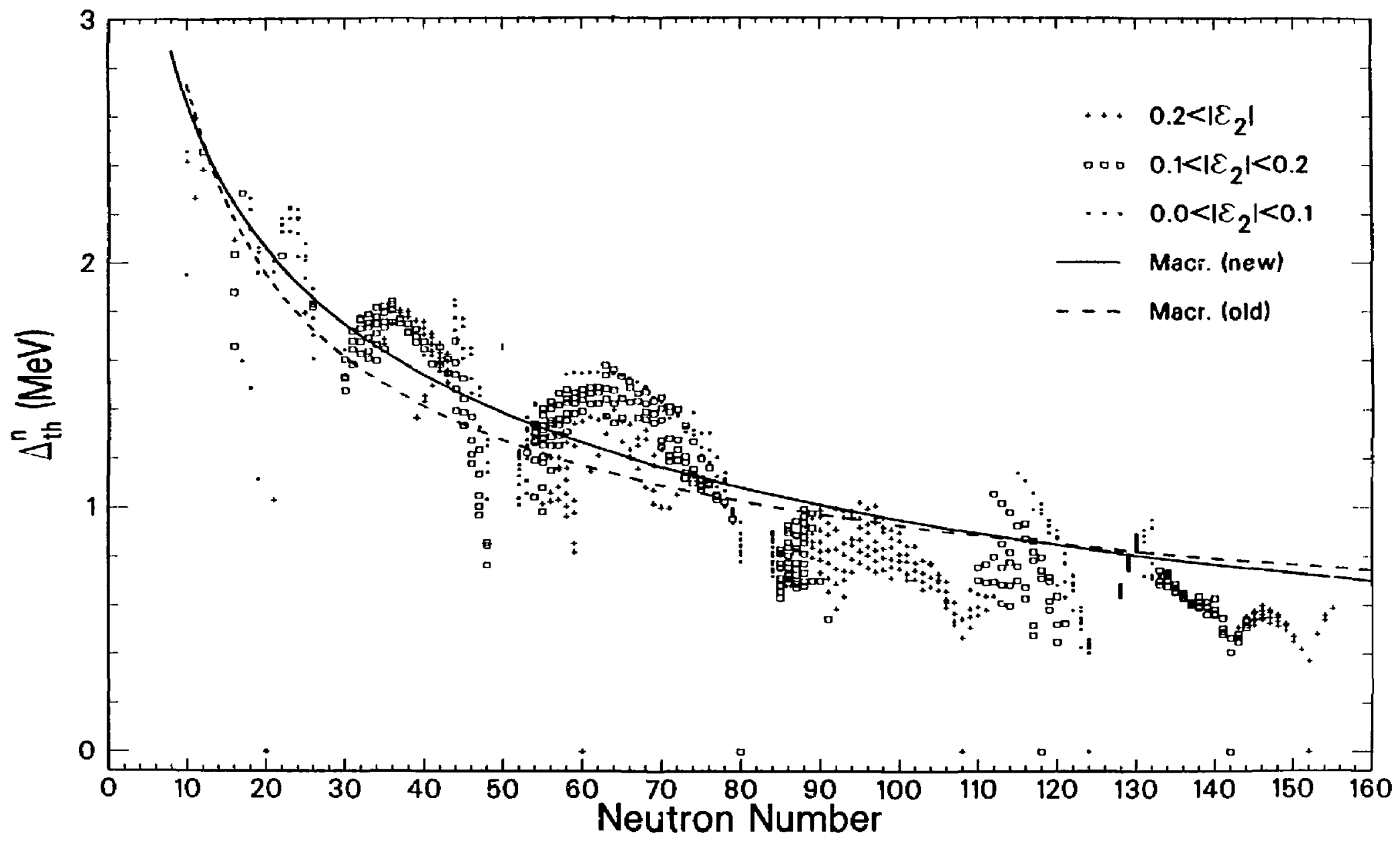

Fig. $2 b$ 


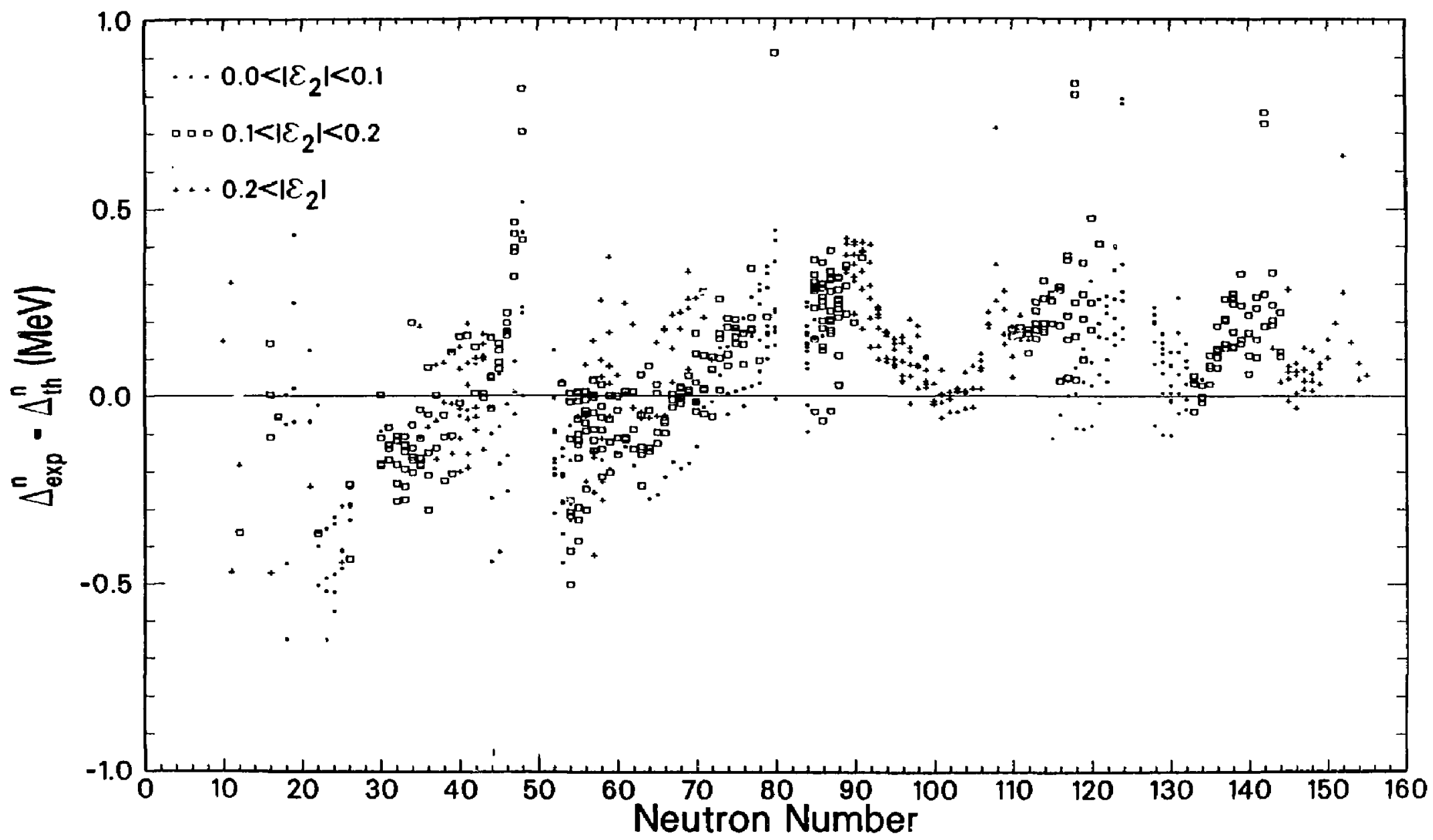

$F_{i g .}{ }_{2 c}$ 